\title{
Food webs: reconciling the structure and function of biodiversity
}

\author{
Ross M. Thompson ${ }^{1}$, Ulrich Brose ${ }^{2}$, Jennifer A. Dunne ${ }^{3,6}$, Robert O. Hall Jr. ${ }^{4}$, \\ Sally Hladyz ${ }^{1}$, Roger L. Kitching ${ }^{5}$, Neo D. Martinez ${ }^{6}$, Heidi Rantala ${ }^{7}$, \\ Tamara N. Romanuk ${ }^{8}$, Daniel B. Stouffer ${ }^{9,10}$, and Jason M. Tylianakis ${ }^{10}$
}

${ }^{1}$ Australian Centre for Biodiversity and School of Biological Sciences, Monash University, Clayton 3800, Australia

${ }^{2}$ Department of Biology, Georg-August University Göttingen, Germany

${ }^{3}$ Santa Fe Institute, Santa Fe, New Mexico, USA

${ }^{4}$ Department of Zoology and Physiology, University of Wyoming, Laramie, Wyoming, USA

${ }^{5}$ Environmental Futures Centre, Griffith University, Nathan, Queensland, Australia

${ }^{6}$ Pacific Ecoinformatics and Computational Ecology Lab, Berkeley, California, USA

${ }^{7}$ Department of Zoology, Southern Illinois University Carbondale, Carbondale, Illinois, USA

${ }^{8}$ Department of Biology, Dalhousie University, Halifax, Nova Scotia, Canada

${ }^{9}$ Integrative Ecology Group, Estación Biológica de Doñana (EBD-CSIC), Sevilla, Spain

${ }^{10}$ School of Biological Sciences, University of Canterbury, Christchurch, New Zealand

The global biodiversity crisis concerns not only unprecedented loss of species within communities, but also related consequences for ecosystem function. Community ecology focuses on patterns of species richness and community composition, whereas ecosystem ecology focuses on fluxes of energy and materials. Food webs provide a quantitative framework to combine these approaches and unify the study of biodiversity and ecosystem function. We summarise the progression of foodweb ecology and the challenges in using the food-web approach. We identify five areas of research where these advances can continue, and be applied to global challenges. Finally, we describe what data are needed in the next generation of food-web studies to reconcile the structure and function of biodiversity.

\section{Reconciling the study of biodiversity and ecosystem function}

We are experiencing two interrelated global ecological crises. One is in biodiversity, with unprecedented rates of species loss across all major ecosystems, combined with greatly accelerated biotic exchange between landmasses [1]. Consequently, spatial and temporal patterns of species occurrence are being fundamentally altered by extinction and invasion. The second crisis concerns the regulation of ecological processes and the ecosystem services they provide. Processes such as primary production and nutrient cycling have been severely altered by human activities [1]

Our understanding of biodiversity comes largely from a sound theoretical and empirical basis provided by community ecology, including core concepts such as niche segregation [2] and Island Biogeography [3,4]. Early studies of individual species' habitat preferences and physiological tolerances have been complemented by studies of interspecific interactions from field surveys and experiments. Applications such as conservation management depend

Corresponding author: Thompson, R.M. (ross.thompson@monash.edu). largely on mapping of community patterns and studies of habitat occupancy and preferences. More recently, habitat models have been applied, and the advent of simple and cheap molecular markers has allowed quantification of important community assembly (see Glossary) processes such as dispersal [5]. In community ecology the unit of study is the individual, population, or species, consistent with other sub-disciplines such as behavioural and population biology.

\footnotetext{
Glossary

Assembly: the set of processes by which a food web is rebuilt after disturbance or the creation of new habitat.

Bioenergetics: the flow and transformation of energy in and between living organisms and between living organisms and their environment.

Cascade model: a food-web model which assumes hierarchical feeding along a single niche axis, with each species allocated a probability of feeding on taxa below it in the hierarchy.

Community web: a food web intended to include all species and trophic links that occur within a defined ecological community. 'Species' in this sense might involve various degrees of aggregation or division of biological species.

Compartmentalisation: the property by which one subset of species within a food web operates, to varying degrees, independently of other parts.

Ecological stoichiometry: how the balance of multiple chemical elements within organisms influences their interactions in food webs.

Ecosystem function: the physical, chemical, and biological processes or attributes that contribute to the self-maintenance of the ecosystem; including energy flow, nutrient cycling, filtering, buffering of contaminants, and regulation of populations.

Interaction strength: a measure of how much a predator alters population size, biomass, or production of its prey. In food webs these are associated with energy flows while noting that predators can affect prey non-trophically.

Niche model: a food-web model which assigns each consumer a feeding distribution on the niche axis that can overlap with itself (cannibalism) and permits trophic loops but still generates realistic patterns whereby species tend to feed in a linear hierarchy.

Quantitative food web: a food web where the interaction strengths or trophic flows are quantified.

Scale-independence and/or dependence: the degree to which attributes of a food web change with food-web size.

Stability: measures of the ability to and/or speed with which a food web regains its structure following a disturbance (resilience) or resists change in
} food web structure (resistance). 
Ecosystem ecology focuses on the fluxes of energy and nutrients through ecological systems [6,7]. Whereas community ecology tends to be reductionist, concentrating largely on processes driven by individuals, populations, or species, ecosystem research often takes a more holistic, systems approach. It remains tractable by aggregating species into broad functional compartments such as primary producers, herbivores, and carnivores, with fluxes of energy and materials between them. This approach has allowed development of flux models, which have been applied widely in forestry, fisheries, and agriculture. However these system-based approaches lack the detail to detect changes in single species, and the compartments used cannot be readily reconciled with other areas of biology [8].

A gap exists between community ecology, which incorporates species diversity, and ecosystem ecology, which can describe changes in function but does not incorporate diversity (Table 1). In the 1980s biodiversity-ecosystem function (BEF) studies attempted to bridge this gap. These studies initially focused on concerns over the consequences of species loss for ecosystem productivity and stability [9]. Early studies relating biodiversity to functions such as primary productivity, decomposition, pollination, and fisheries production were necessarily simple and primarily focused on single trophic levels ([9] for a review). The limitations of this approach have been widely described $[9,10]$, and there is a growing recognition that BEF studies would benefit from a framework that considers effects of changes in biodiversity across trophic levels on multiple ecosystem processes [9-11].

We propose that reconciling biodiversity and ecosystem function in a single conceptual framework is best achieved through application of a food-web approach. Food webs are maps of the trophic interactions between species, usually simplified into networks of species and the energy links between them. These networks have a suite of attributes which can be calculated to describe food web structure (Box 1). Because this approach includes both species and energy flows among species, food webs provide a natural framework for understanding species' ecological roles and the mechanisms through which biodiversity influences

Table 1. An overview of community ecology and ecosystem science, and the potential for food-web ecology to integrate across disciplines

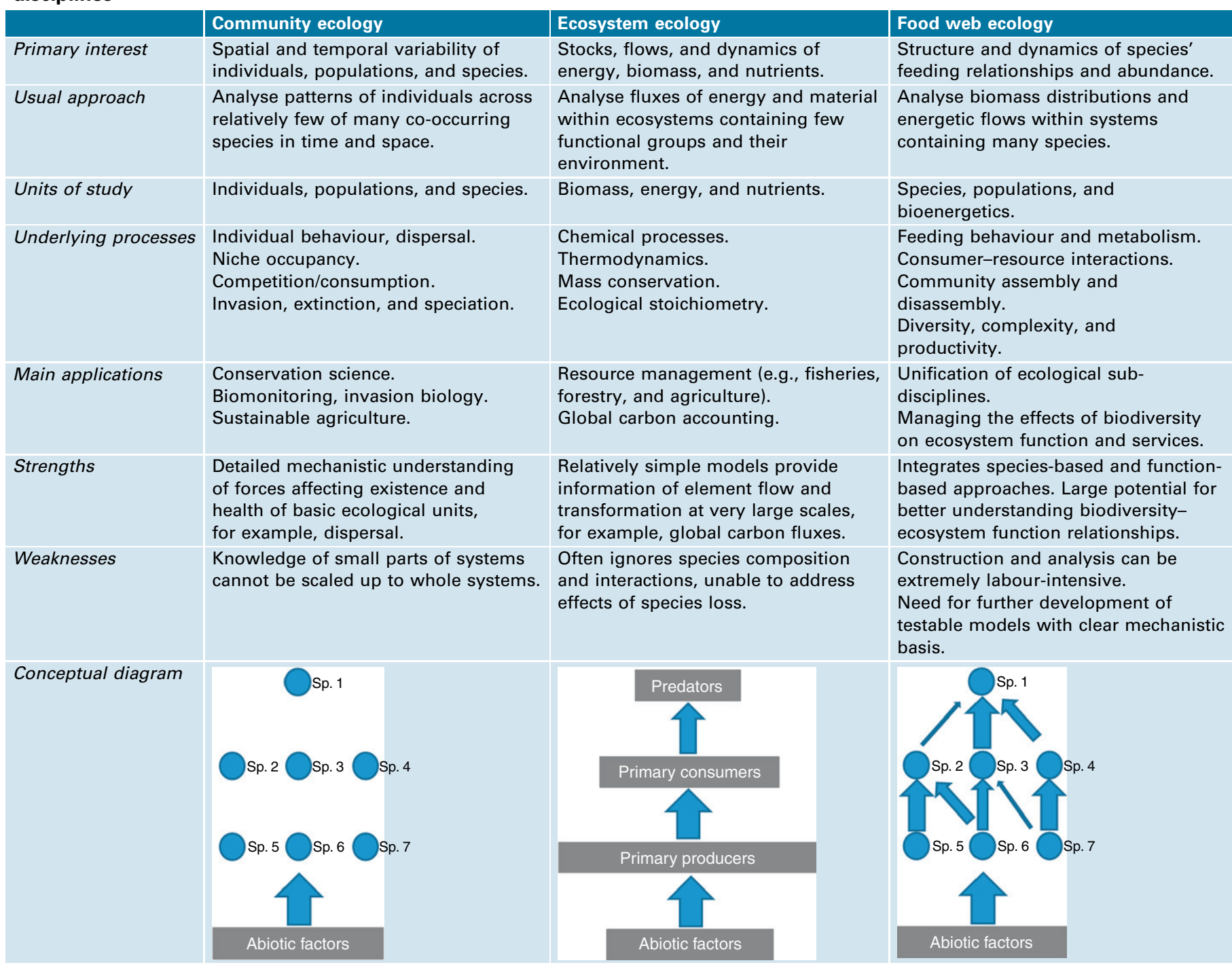




\section{Box 1. Food webs and food-web attributes}

Food webs characterise the networks of trophic interactions that occur among species within ecological communities. We focus here on biodiversity and ecosystem function of whole communities of species rather than more narrowly defined networks such as host-parasitoid and mutualistic networks ${ }^{5}$. Individual species, aggregates of species, life-stages of species, or non-taxonomic groups (e.g., detritus) form nodes within food webs. Flows of energy form links via transfers of live or dead biomass between nodes. Food webs are usually characterised as binary networks where links are either present or absent, although webs with weighted links that quantify energy flows ('weighted networks') are becoming increasingly common [18,90]. Many attributes are used to describe aspects of food-web structure (Table I).

Table I. Food webs

\begin{tabular}{|c|c|}
\hline Food-web attribute & Biological meaning \\
\hline Taxa richness (S) & Number of taxa (nodes) in the food web. \\
\hline Number of trophic links (L) & Number of directed feeding links (edges) between taxa. \\
\hline Linkage density (= L/S) & Number of links per taxon. A measure of mean dietary specialisation across the food web [90]. \\
\hline Connectance $(\mathrm{C})\left(=\mathrm{L} /\left\{\mathrm{S}^{2}\right\}\right)$ & $\begin{array}{l}\text { Proportion of potential trophic links that do occur. An indication of degree of inter-connectivity in a food } \\
\text { web, typically } 0.05-0.30[91,92] \text {. }\end{array}$ \\
\hline Generality (G) & The mean number of prey per consumer [93]. \\
\hline Vulnerability (V) & Mean number of consumers per prey [93]. \\
\hline Food chain & $\begin{array}{l}\text { A distinct path within the food-web matrix from any taxon down to a basal taxon (a taxon which feeds on no } \\
\text { other taxa) [18]. }\end{array}$ \\
\hline Mean chain length (mean $\mathrm{FCL}$ ) & $\begin{array}{l}\text { Average number of links found in a food chain across a food web [94]. Food-chain length appears to be } \\
\text { reduced by disturbance and increased by higher energy supply and increased ecosystem size [21-23]. }\end{array}$ \\
\hline Maximum chain length (max $\mathrm{FCL}$ ) & The maximum number of links found in any food chain in a food web [94]. \\
\hline Number of basal taxa (b) & The number of taxa which do not consume any other taxa, by definition autotrophs. \\
\hline Number of intermediate taxa (i) & The number of taxa which are both consumed by, and consume, other taxa. \\
\hline Number of top taxa $(t)$ & The number of taxa which are not consumed by any other taxa. \\
\hline Prey:predator $(=\{b+i\} /\{t+i\})$ & $\begin{array}{l}\text { A measure of food-web 'shape'; high values are more triangular, low values are more 'square' in shape. } \\
\text { When }<1 \text { the food web has an inverted structure that might indicate instability. Note criticisms of this } \\
\text { attribute [95] and its sensitivity to the common practice of aggregating of low trophic level taxa. }\end{array}$ \\
\hline Robustness & $\begin{array}{l}\text { The minimum level of secondary extinction that occurs in response to a particular perturbation (species } \\
\text { removal) [96]. }\end{array}$ \\
\hline Food-web motifs & $\begin{array}{l}\text { The set of unique connected parts of a food web containing } n \text { species. Can be thought of as the fundamental } \\
\text { building blocks of complex networks. Most studies focus on triplets of species, for which there are } 13 \\
\text { possible combinations. }\end{array}$ \\
\hline Degree distribution & $\begin{array}{l}\text { The frequency distribution of the number of interactions per taxa (termed its 'degree'). Can identify } \\
\text { important interactors such as keystone species. }\end{array}$ \\
\hline Intervality & $\begin{array}{l}\text { The degree to which the prey in a food web can be ordered so that the diets of all species are placed } \\
\text { contiguously within a single dimension [82]. }\end{array}$ \\
\hline
\end{tabular}

ecosystem function [12]. Recognising that the relationship between biodiversity and ecosystem function is a reciprocal one, we have taken an inclusive approach, and consider mechanisms whereby biodiversity can influence function, and function can influence biodiversity. We have deliberately not considered stability as a function, as this has been the subject of a recent major review [13]. We use five core challenges for the application of food-web approaches to biodiversity and ecosystem function studies to organise our discussion of emerging trends in this area. We finish by summarising those trends into an overview of what the next generation of food-web studies needs to include in order to be applied to the study of biodiversity and ecosystem function.

\section{Food webs as a tool for BEF studies}

The use of food webs to describe fluxes of energy between species was mired for many years in debate over inclusiveness, approaches to sampling, and the meaning of some of the food-web attributes that can be calculated [13-15]. There is now a clear understanding of the limitations of older data and the need for methodological rigour in describing food webs [14,15]. With those concerns dealt with, food-web ecology can be placed in the context of other major sub-disciplines of ecology. Table 1 shows the potential for food-web ecology to act as an underlying conceptual and analytical framework for studying biodiversity and ecosystem function. However, doing so requires addressing a series of key challenges.

\section{Challenge one: relating food-web structure to ecosystem function}

There is now an established suite of attributes that describe food-web structure, and it is known that food-web structure can influence function $[16,17]$. The central challenge is determining which aspects of structure are related to which aspects of function. Because many food-web attributes systematically vary with the size and complexity of webs (Box 1, [18,19]) understanding these underlying patterns is critical in determining relationships between attributes and ecosystem functions.

Simple relationships between food-web attributes and ecosystem functions Traditionally, most food webs have been described as 'binary' networks, with trophic links between taxa identified as either present or absent. These relatively simple representations of food webs allow calculation of a suite of attributes that can be correlated with measures of ecosystem function. The basic attribute of food-web size is measured as the number of species $(S)$. Complexity measures incorporate the number of trophic links $(L)$ in terms of link density $(L / S)$ or connectance, the 
proportion of potential links that actually occur $\left(L / S^{2}\right)$. A central measure of all networks' structure is the variability of links among nodes or 'degree distributions' that, in food webs, describe the balance among trophic specialists and generalists. When normalized by $L / S$, degree distributions have a general exponential-type shape [20] indicating that most paths of energy flow through food webs go through relatively few species. This is consistent with species removal experiments in BEF studies, which have suggested a small sub-group of species disproportionately influences productivity [9].

Incorporating energy flow Weighted networks allow foodweb metrics to include the strength of trophic interactions, and therefore provide an estimate of energy flow through the web. Relationships between energy fluxes and biodiversity have been proposed in the past, notably that systems with larger amounts of energy entering the food web should be able to support longer food chains and hence more biodiversity [6,21], although there is the potential for interactions with ecosystem size and disturbance $[22,23]$. The power of integrating ecosystem functions into food-web studies is clearly illustrated by the studies that have related foodchain length to basal energy supply. Importantly these are also readily applicable to management issues. For instance, food-web studies have shown that riparian vegetation influences energy inputs to streams, with consequences for fish populations [24]. A potential application is using basal productivity data and food-web models to determine the size of reserves needed to support top predators.

Emerging trends and future directions There is considerable potential to use food webs as a tool for achieving greater understanding of the relationships between attributes of food webs and ecosystem function. As yet there have been relatively few studies of the effects of food-web attributes on various ecosystem functions in a multi-trophic level context. Studies of variability in food web attributes along productivity gradients have suggested that such relationships do exist [21], but there is a need for experimental studies to explore the underlying mechanisms. Most importantly, understanding the reciprocal nature of relationships between food web structure and ecosystem function is essential. This understanding will only come from manipulative studies of food webs where both species composition and ecosystem functions are measured and manipulated individually and in combination.

New measures of structure of food webs also have potential to show correlations with function [18]. A foodweb motif is a recognisable regular pattern of connections between nodes. Using food-web motifs to decompose larger, more complex food webs into more basic building blocks $[25,26]$ shows that motifs are shared by food webs from distinct habitat types [26]. Motif analyses has also quantified the 'embeddedness' of individual species within their network in order to better link to traditional community ecology concepts such as keystone species [27]. These relationships underpin food-web function - in particular the dynamics of food webs when subjected to extinctions, invasions, and other disturbances. Fortunately, such modelling is becoming increasingly tractable.

\section{Challenge two: combining food web and ecosystem models}

Biodiversity studies typically describe species' occurrence and abundance distributions at one or a small number of points in time. In contrast, ecosystem studies typically describe fluxes of energy and materials on longer time scales (years to decades). This increased temporal resolution of ecosystem studies combined with more focus on quantifying energy flows forced ecosystem ecologists to simplify systems into relatively few compartments. Early species-based dynamical food-web modelling also used simplifications. May [28] and others conducted local stability analyses for diverse communities at equilibrium, using interaction matrices where the species were linked randomly and had random interaction strengths. Other researchers used non-linear dynamical approaches, but only for small modules of interacting species [29]. Understanding BEF relationships requires dynamic food-web models with a full suite of interacting species and incorporating fluxes of materials.

The cascade and niche models of food webs One important step in modelling the dynamics of complex networks has been introducing realistic, non-random underlying structure. In the 1980s, Joel Cohen and colleagues developed the hierarchical cascade model [30], which assumes hierarchical feeding along a single niche axis, with each species allocated a probability of feeding on species below it in the hierarchy. The cascade model did a reasonable job of generating structure similar to the empirical datasets used at the time, but did poorly with more realistic, complex datasets, and failed to incorporate some natural features such as trophic loops (where Species A consumes Species B, which consumes Species $\mathrm{C}$ which consumes Species A) and cannibalism.

The niche model [31] modifies the cascade model by assigning each consumer a feeding distribution on the niche axis that can overlap with itself (cannibalism), and permits trophic loops. The niche model matches empirical data much better than the cascade or random models, and a probabilistic version of the niche model is providing ways to further explore the extent to which the constraints imposed by the niche model are consistent with empirical data [32]. These simple models allow exploration of how food-web structure systematically depends on the number of links and species in a system [20].

Incorporating ecosystem function into food web models Modelling BEF relationships requires realistic dynamic food web models as a basis on which are superimposed fluxes of materials. The niche model has become one of the main tools for providing the underlying food-web structure on which complex bioenergetic models are run, allowing modelling of the relationships between biodiversity and ecosystem function. Such models can now simulate the biomass dynamics of 50 or more interacting species. This means that it is possible to model how factors such as the functional responses in consumer-resource interactions [33], adaptive consumer behaviour [34], and body-size distributions across species [35] influence biodiversity in complex communities. For example, in multi-trophic level food webs where coexistence of primary producers is strongly 
limited by competition for abiotic resources, dynamic models demonstrated that top-down control is necessary to prevent competitive exclusion and thus species loss [35]. These dynamic models predict species interactions by the body sizes of the taxa using empirically well-supported allometric scaling relationships of functional attributes such as metabolic and feeding rates with body sizes. This type of dynamical modelling is now being applied to specific systems, such as time-series data for 24 guilds of taxa in Lake Constance, northern Europe [36]. This marks the first time that an ecosystem model has successfully reproduced the well-resolved seasonal plankton succession of a lake, and represents an important step towards using such models to improve conservation and management decisions. For example, such a model could be used to assess the effects of changes in nutrient loads on plankton communities and therefore whether the lake may be prone to algal blooms.

Emerging trends and future directions The development of models that use underlying food-web models as a framework for modelling patterns of body mass and body size are the first step towards predicting patterns of energy flow. Because most physiological functions scale predictably with body size, the ability to incorporate this variable into food web models is a critical step. For example in spring-fed creeks, changes in body size result in changes in energy flow via differences in size-dependent consumption and processing rates [37]. Food web models that incorporate body size will allow prediction of the knock-on effects of changes in body size for total fluxes of energy through food webs, and any indirect effects on other taxa. Such models are likely to be very powerful, particularly given predicted and experimentally-demonstrated effects of climate change on body size, species abundances, and prey selection in food webs [38], which have currently unknown effects on ecosystem function.

\section{Challenge three: from individual traits to ecosystem function}

Relating the traits of individual organisms to their ecological functions is a significant challenge. Individual variation in behaviour and physiology can cascade up to ecosystem processes [39], and adaptive behaviour can be an important force structuring food webs [34]. Although food webs ideally integrate ecological levels from individuals to populations to ecosystems, most work has occurred at the population level. Much recent effort has focused on extending predator-prey population dynamics to food webs, and has shown emergent food-web attributes, which result from interactions of population-level dynamics [34,35]. Even more complete models that include individual variability in consumption and competition and incorporate evolutionary dynamics have now been developed [40]. These models can predict energy flow and biomass for relatively small food webs, but have the potential to be extended to larger food webs.

Linking individuals to food webs to ecosystem function Research on metabolic drivers of individuals' consumerresource interactions provides a powerful way to understand BEF relationships [41]. Metabolism depends systematically on body size and environmental temperature [42]. Extending these simple physiological relationships for individual animals to the level of whole communities can be achieved using relatively simple foodweb models. By assuming equilibria between metabolic demands and resource supply through the links, mass balance models can estimate energy fluxes through food webs. Recently, studies of how body masses and associated energy fluxes are distributed across trophic levels [43] have shown how non-random distributions of species' interaction strengths constrain complex food webs into quantitative configurations, preventing unstable consumer oscillations and competitive exclusion among basal species [40]. Food webs in this context have provided a framework for bringing together individual physiology, community energy flux, and stability to predict relationships between species diversity and ecosystem functioning [44].

Emerging trends and future directions Increasingly, individual-level variability is being incorporated into food web studies, and applied to understanding BEF relationships. This has been aided by novel approaches to the partitioning of variation in empirical studies [45]. Incorporating individual variability in body size of animals into food web models has already been discussed above. However, individual-to-individual variability in resource use can also be seen in a number of groups, including individual niche specialists in trees [46] and individual 'specialists' and 'generalists' within rodent populations [47]. Incorporating these complexities into food web models will be challenging, but there are clear avenues for future research. Individualbased modelling (IBM) approaches have the potential to bring individual-level variability into food-web models $[48,49]$. Increases in computational power are now sufficient to support IBM approaches to connect individual-level process with population-level structure and dynamics in food webs [48] but as yet have been little used in this context.

\section{Challenge four: incorporating space and time into BEF studies}

Spatial and temporal variability in food web structure has the potential to influence ecosystem function and all of the underlying relationships so far discussed.

\begin{abstract}
Spatial variability in food web structure and ecosystem functions Most food webs do not include spatial variation of web structure. Empirical studies incorporating spatial variability have shown that food-web topology is strongly influenced by spatial segregation of taxa [50]. Theoretical studies have long shown the potential for spatial variability and movement of animals to stabilize food-web dynamics [51,52]. More recently, empirical studies have shown the potential for spatial coupling across food webs based on an animal's adaptive foraging behaviour [53]. The movement of predators between patches of resources can be a critical component in determining landscape-scale persistence of taxa and landscape-scale maintenance of ecosystem functions [54].
\end{abstract}

Temporal variability in food web structure and ecosystem functions Temporal variability also remains a poorlyunderstood feature of food-web dynamics. Several studies 
have shown that intra-annual temporal variability alters food-web structure $[55,56]$, but the interaction between temporal variability and ecosystem functions remains unclear. Because organisms at higher trophic levels tend to be larger and longer-lived, the temporal scale of response becomes longer as we move up food webs. This means that predator responses to change lag behind those of primary consumers. Increasing rates of biological change, such as those occurring due to climate change, might therefore alter food-web dynamics, favouring bottom-up forces, increasing biomass of basal groups such as plants, algae, and bacteria [57]. Food webs have already provided a conceptual framework for generating hypotheses on the effects of changing climate [58]. There is clear evidence from the empirical studies to date that the functional consequences of climate change can be predicted, modelled, and understood using food-web approaches [59,60].

At much longer temporal scales, evolution is an active force within food webs, because trophic relationships are a product of phylogenetic relationships and evolutionary relationships between predators and prey [61,62]. The interplay between intraspecific (e.g., closely related taxa tend to share trophic relationships) and inter-specific (e.g., strong interactions between predators and prey, and plants and pollinators) interactions will have profound effects on food-web structure [63], and the balance of these can determine the occurrence and strength of niche partitioning effects on ecosystem functioning [9]. Most studies have considered food webs as a fixed suite of species with no variation in patterns of connectivity through evolutionary time [64]. As a result, speciation within food webs remains poorly understood. Even a model with small numbers of species and relatively few traits shows that many food-web outcomes are possible [65]. A number of recent reviews have discussed the potential for food webs to influence speciation, and therefore ecological functions $[66,67]$, and this topic will not be covered in detail here.

Emerging trends and future directions The increasing ability to model temporal variability in food-web structure and thus ecosystem function using dynamic models has been discussed above. There is considerable potential to further extend the temporal nature of those models to include evolutionary processes. It is surprising that there have not been similar attempts to incorporate spatial variability into models of food web structure. As spatial ecology increasingly considers ecological processes in a landscape framework, there is potential to develop metafood-web theory to mirror developments in meta-community and meta-ecosystem theory. This needs to include the movements of individuals - as the movement of top predators (in particular) across landscapes can strongly affect local food web structure and ecosystem function [68]. However, there remains a gap between general ecological theory in this space, food web ecology, and ultimately BEF theory. The Unified Neutral Theory of Biodiversity [69] has made a major contribution to spatial ecology over the last decade. However, the theory has features which make it incompatible with food webs, including a core assumption of ecological equivalence and an inability to incorporate trophic structure. Developing theory and models which reconcile neutral type dynamics and food web models is a key challenge for the next decade.

\section{Challenge five: biodiversity loss and effects on ecosystem function}

Predicting biodiversity using food web models Previous research on the relationship between biodiversity and ecosystem functioning has been criticised for its assumption of random species losses in experiments because extinctions are known to occur non-randomly in nature [70]. Predicting which species will go extinct has been a major research avenue in food-web ecology, which could inform future biodiversity experiments. Simulations based on network structure use random or systematic removal of certain species from empirical food webs can predict which species are left without interacting partners and would thus go secondarily extinct [71]. Dynamical models are also being used to explore primary and secondary species extinctions [72,73]. The position of species in a network has been used to estimate their dynamic importance [27] and vulnerability to extinction [74]. In an applied context, food-web studies have now been used to determine future vulnerability to species loss. Using food-web analysis of food webs degraded by over-fishing, it was possible to show that the degraded food webs were more vulnerable to future species loss than comparable marine food webs which had been subjected to lower levels of exploitation [75].

Emerging trends and future directions Understanding how the locations of species within a network relate to their functional role has the potential to provide a major advance in BEF research. The vast majority of studies predicting effects of species loss on biodiversity are based on single trophic levels, meaning that adopting a food web approach could fundamentally alter expectations of the effects of extinctions [76]. Food-web models already exist that can predict potential secondary extinctions, but these have not been extended to predict functional consequences of species loss. This predictive ability may be particularly important when it is considered that extinctions within food webs are highly non-random with respect to functional roles [77-79]. Mapping functional roles onto vulnerability to primary and secondary extinctions in food webs is a highly promising area of future research.

Food webs are also now at the point where we understand what attributes reliably reflect degradation and vulnerability to future change. For example, marine food webs that have been degraded have simpler food web structure (shorter food chains, less trophic loops, and lower connectivity), with likely consequences for food web stability [75]. Food webs have not yet been used as indices of degradation, in a systematic sense. However, particularly in marine environments, we are well positioned to apply food webs in this way [80].

\section{Concluding remarks}

Achieving a mechanistic understanding of how biodiversity relates to ecosystem function is necessary for estimating the impacts of species loss and invasion, and in managing ecosystems for ecosystem services [10]. The 
BEF relationship within trophic levels is underpinned by niche partitioning, which is implicit in the niche model of food-web structure [81]. Food-web research has made considerable progress in developing metrics for intervality, which describe resource partitioning and directly relate species loss to occupation of niche space $[82,83]$.

Food-web approaches emphasise that biodiversity is not only an independent variable within BEF research, but also a dependent variable in terms of an ecosystem's ability to maintain biodiversity. Attributes of food-web structure, such as allometric degree distributions [43], appear to be critical for biodiversity maintenance. Current research challenges include linking specific structural and functional attributes of food webs and their biotic and abiotic context to the maintenance of specific components of biodiversity (e.g., genetic, species, and interaction diversity), and ecosystem function (e.g., total trophic flow and biomass maintenance). Recent advances in the quantification of multivariate functional traits of species [84] need to be integrated into network analyses to generate a more mechanistic approach to biodiversity and ecosystem functioning based on species traits, interactions, and energy flow.

Using food webs, we can extend the attributes of individuals and populations to ecosystem properties such as production and element cycling. Earlier modelling approaches explored the impacts of trophic dynamics on ecosystem outcomes [85] in experimental contexts. Future dynamical modelling will explore impacts of population dynamics on functions such as ecosystem-level production and throughput, and applied to questions of how anthropogenic changes will affect the functioning of ecosystems.

Food webs have provided a framework for the integration of diverse sub-fields of ecology. Food-web structure is well described, the significance of numerous food-web attributes is understood, and there exist sophisticated and useful modelling approaches. In this context, there are clear questions that now can be addressed in food-web ecology. Describing how food web topology changes along environmental gradients such as eutrophication or physical disturbance [86,87] and over time in heavily impacted systems is the first step [75]. Linking these topological changes to changes in ecosystem functioning and the robustness of systems to further change is a major challenge. However, it is exactly for challenges such as these where food-web approaches hold the most promise. Invasion-extinction dynamics are most profitably viewed through a lens of trophic change, as it not just biodiversity change that is important but the ecosystem-wide changes in functioning that accompany changes in the identities of the players. Observations of the 'invasional meltdown' of communities and related ecosystem functions on Christmas Island, after the arrival of the yellow crazy ant, provide an illustration of the utility of considering systems in this way [88].

Can our existing knowledge be applied to understanding $\mathrm{BEF}$ relationships in a management context? Research on the interplay between food-web structure and ecosystem function can provide managers with a powerful tool to understand, predict, and manage ecosystems experiencing multiple competing demands and environmental perturbations. In some cases a food-web approach has already been effectively applied: for instance in marine fisheries management $[75,80]$ and in studies of functional consequences of anthropogenic impacts on stream and river ecosystems [89] (Box 2).

The development of quantitative analysis of food webs provides an opportunity to reconcile ecological processes and biodiversity patterns into a single analytical framework. Importantly this is increasingly coupled with the ability to model the effects of food-web structure on ecosystem functions. While the data we have can provide insights into these relationships, the next generation of biodiversity

\section{Box 2. A case study - food-web insights from the Colorado River}

Food-webs can elucidate ecological responses to management actions. Cross et al. [89] studied the effects of an experimental flood in the Colorado River, by describing the full food web of the ecosystem, including energy flows from primary producers to animals; invertebrate community composition, biomass, and production; and fish biomass and production (Figure I). The flood reduced production of invertebrate biota considerably, but highly nonrandomly; two dominant invasive invertebrates, Potamopyrgus antipodarum and Gammarus lacustris declined substantially. Counter-intuitively, the biomass of rainbow trout (Oncorhynchus mykiss)

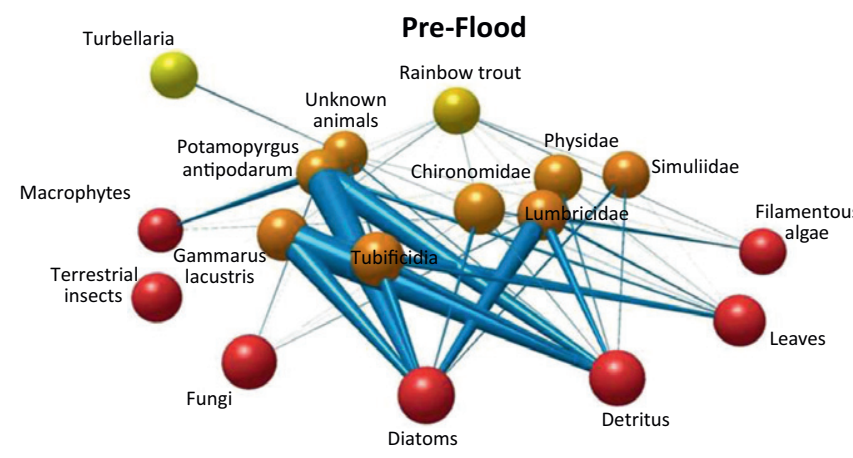

increased after the high flow. Crucially, understanding the patterns of energy flow in a food-web context allowed the complex cause and effect relationships of the experimental flood to be disentangled. Post-flooding, the production of two sub-dominant but palatable invertebrate taxa (Simuliidae and Chironomidae) increased greatly. Because these invertebrates had higher interaction strengths with rainbow trout, energy flow to trout increased, leading directly to the observed increases in trout biomass. Here, using food-web approaches allowed a clear understanding of what was influencing a functional outcome of management interest (trout biomass).

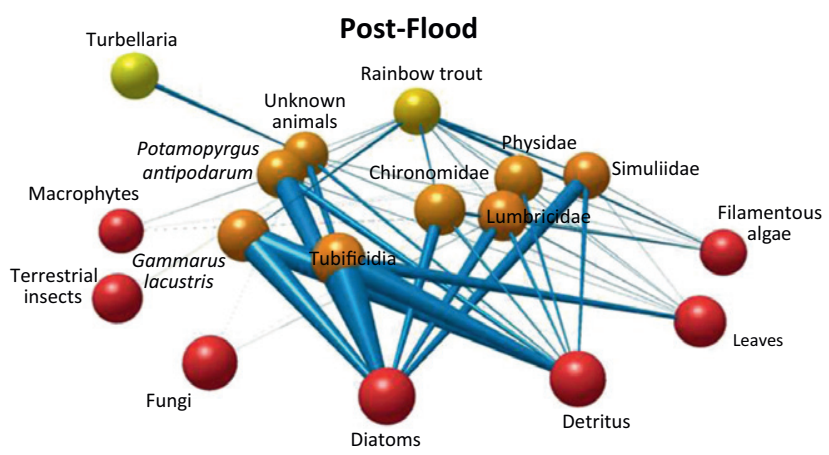




\section{Box 3. Taking a food-web approach to reconciling the structure and function of biodiversity}

The next generation of empirical studies addressing the relationships between biodiversity and ecosystem function can benefit from adopting a food-web approach. Such studies need to have the following characteristics.

Diversity within functional groups needs to be explicitly measured, particularly in ecosystem ecology studies. This can be carried out either through traditional taxonomy or using molecular approaches. Where molecular data is available, phylogenetic approaches can be applicable.

Fluxes of energy and materials need to be incorporated, particularly in community ecology studies. While measurements of interaction strengths can be needed, binary representations of links are also informative. Methods for measuring primary and secondary production need to be incorporated into food web studies. There is considerable potential for stable isotope approaches and related mixing models to be used as a means to assess food sources of animals and the origin of nutrients for primary producers.

Incorporation of information on species traits such as body size and physiological traits. These traits are likely to influence both ecosys-

and ecosystem function studies need to adopt a food-web approach (Box 3) in order to disentangle the complex interrelationships between pattern and process in ecological systems.

\section{Acknowledgements}

The GlobalWeb retreat (Melbourne 2011) was fully funded by Monash University via a Monash Research Accelerator grant to Ross Thompson. Ross Thompson is supported by an Australian Research Council Future Fellowship (FT110100957). Jennifer Dunne and Neo Martinez were supported by NSF DBI-1048302. Ulrich Brose was supported by the German Research Foundation (BR 2315/13), and Jason Tylianakis is supported by a Rutherford Discovery Fellowship. Daniel Stouffer was supported by a Juan de la Cierva Fellowship from the Ministerio de Ciencia e Innovación (MICINN), Spain. Tamara Romanuk is supported by the National Sciences and Engineering Research Council of Canada (NSERC). This paper was greatly improved through the comments of Guy Woodward, Paul Craze, and three anonymous reviewers.

\section{References}

1 MEA (2005) Millennium Ecosystem Assessment, Synthesis Report

2 Hutchinson, G.E. (1957) Population studies - animal ecology and demography - concluding remarks. Cold Spring Harb. Sym. Quant. Biol. 22, 415-427

3 Macarthur, R.H. and Wilson, E.O. (1963) Equilibrium-theory of insular zoogeography. Evolution 17, 373-387

4 Hubbell, S.P. (2001) The Unified Neutral Theory of Biodiversity and Biogeography, Princeton University Press

5 Shea, K. et al. (1998) Management of populations in conservation, harvesting and control. Trends Ecol. Evol. 13, 371-374

6 Lindeman, R.L. (1942) The trophic-dynamic aspect of ecology. Ecology 23, 399-418

7 Pace, M.L. and Groffman, P. (1998) Successes, Limitations, and Frontiers in Ecosystem Science, Springer

8 Loeuille, N. and Loreau, M. (2005) Evolutionary emergence of sizestructured food webs. Prco. Natl. Acad. Sci. U.S.A. 102, 5761-5766

9 Hooper, D.U. et al. (2005) Effects of biodiversity on ecosystem functioning: a consensus of current knowledge. Ecol. Monogr. 75, 3-35

10 Montoya, J.M. et al. (2003) Food web complexity and higher-level ecosystem services. Ecol. Lett. 6, 587-593

11 Cardinale, B.J. et al. (2009) Separating the influence of resource 'availability' from resource 'imbalance' on productivity-diversity relationships. Ecol. Lett. 12, 475-487

12 Thebault, E. and Loreau, M. (2003) Food-web constraints on biodiversity-ecosystem functioning relationships. Proc. Natl. Acad. Sci. U.S.A. 100, 14949-14954

13 Rooney, N. and McCann, K. (2011) Integrating diversity, food web structure and stability. Trends Ecol. Evol. 27, 40-46 tem function and trophic interactions. Understanding potential tradeoffs between functional roles and food-web interactions is essential. Consideration of biodiversity and ecosystem function studies in a landscape context. Food-web studies that take into account functional attributes such as dispersal and resource tracking will be able to relate patterns of food-web structure across landscapes to large-scale measures of ecosystem function such as regional productivity.

Use of well understood food-web and network attributes to explore relationships between structure and function. Despite a troubled history, a suite of food-web and network attributes now exist which are likely to have relationships with functional measures. However, there is a need for studies that include both of these factors.

Use of species removal experiments and studies as the basis for dynamic models which include both biodiversity and ecosystem function. Species removal experiments have been frequently carried out, but measuring consequences for food-web structure and ecosystem function is extremely rare. These studies have the potential to provide powerful insights into the functional consequences of invasion and extinction.
14 Dunne, J.A. (2006) The network structure of food webs. In Ecological Networks: Linking Structure to Dynamics in Food Webs (Pascual, M. and Dunne, J.A., eds), pp. 27-86, Oxford University Press

15 Ings, T.C. et al. (2009) Ecological networks - beyond food webs. J. Anim. Ecol. 78, 253-269

16 Strogatz, S.H. (2001) Exploring complex networks. Nature 410, 268276

17 Pascual, M. and Dunne, J.A. (2006) From small to large ecological networks in a dynamic world. In Ecological Networks: Linking Structure to Dynamics in Food Webs (Pascual, M. and Dunne, J.A., eds), pp. 3-24, Oxford University Press

18 Bersier, L.F. et al. (2002) Quantitative descriptors of food-web matrices. Ecology 83, 2394-2407

19 Montoya, J.M. and Sole, R.V. (2003) Topological properties of food webs: from real data to community assembly models. Oikos 102, 614622

20 Stouffer, D.B. et al. (2005) Quantitative patterns in the structure of model and empirical food webs. Ecology 86, 1301-1311

21 Thompson, R.M. and Townsend, C.R. (2005) Energy availability, spatial heterogeneity and ecosystem size predict food-web structure in streams. Oikos 108, 137-148

22 McHugh, P.A. et al. (2010) Dual influences of ecosystem size and disturbance on food chain length in streams. Ecol. Lett. 13, 881-890

23 Post, D.M. et al. (2000) Ecosystem size determines food-chain length in lakes. Nature 405, 1047-1049

24 Kawaguchi, Y. et al. (2003) Terrestrial invertebrate inputs determine the local abundance of stream fishes in a forested stream. Ecology 84, 701-708

25 Bascompte, J. and Melian, C.J. (2005) Simple trophic modules for complex food webs. Ecol. Soc. 86, 2868-2873

26 Stouffer, D.B. et al. (2007) Evidence for the existence of a robust pattern of prey selection in food webs. Proc. R. Soc. B: Biol. Sci. 274, 1931-1940

27 Stouffer, D.B. et al. (2012) Evolutionary conservation of species' roles in food webs. Science $335,1489-1492$

28 May, R.M. (1972) Will a large complex system be stable? Nature 238, 413-414

29 Pimm, S.L. and Lawton, J.H. (1977) Number of trophic levels in ecological communities. Nature 268, 329-331

30 Cohen, J.E. and Newman, C.M. (1985) A stochastic theory of community food webs. I. Models and aggregated data. Proc. R. Soc. B: Biol. Sci. 224, 421-448

31 Williams, R.J. et al. (2010) The probabilistic niche model reveals the niche structure and role of body size in a complex food web. PLOS ONE 5, e12092

32 Williams, R.J. and Purves, D. (2011) The probabilistic niche model reveals substantial variation in the niche structure of empirical food webs. Ecology 92, 1849-1857

33 Williams, R.J. and Martinez, N.D. (2004) Stabilization of chaotic and non-permanent food-web dynamics. Eur. Phys. J. B. 38, 297-303 
34 Kondoh, M. (2003) Foraging adaptation and the relationship between food-web complexity and stability. Science $299,1388-1391$

35 Brose, U. et al. (2006) Allometric scaling enhances stability in complex food webs. Ecol. Lett. 9, 1228-1236

36 Boit, A. et al. (2012) Mechanistic theory and modelling of complex foodweb dynamics in Lake Constance. Ecol. Lett. 15, 594-602

37 Dossena, M. et al. (2012) Warming alters community size structure and ecosystem functioning. Proc. R. Soc. B: Biol. Sci. 279, 3011-3019

38 Sheridan, J.A. and Bickford, D. (2011) Shrinking body size as an ecological response to climate change. Nat. Climate Change 1, 401-406

39 Hawlena, D. and Schmitz, O.J. (2010) Physiological stress as a fundamental mechanism linking predation to ecosystem functioning. Am. Nat. 176, 537-556

40 Shertzer, K.W. et al. (2002) Predator-prey cycles in an aquatic microcosm: testing hypotheses of mechanism. J. Anim. Ecol. 71, 802-815

41 Melián, C.J. et al. (2011) Eco-evolutionary dynamics of individualbased food webs. In Advances in Ecological Research (Vol. 45) (Belgrano, A. and Reiss, J., eds), pp. 225-268, Academic Press

42 Brown, J.H. et al. (2004) Toward a metabolic theory of ecology. Ecology 85, 1771-1789

43 Otto, S.B. et al. (2007) Allometric degree distributions facilitate foodweb stability. Nature 450, U1226-U1227

44 Olff, H. et al. (2009) Parallel ecological networks in ecosystems. Philos. Trans. R. Soc. B 364, 1755-1779

45 Bolnick, D.I. et al. (2002) Measuring individual-level resource specialization. Ecol. Soc. 83, 2936-2941

46 Clark, J.S. (2010) Individuals and the variation needed for high species diversity in forest trees. Science 327, 1129-1132

47 Araujo, M.S. et al. (2010) Nested diets: a novel pattern of individuallevel resource use. Oikos 119, 81-88

48 Grimm, V. (1999) Ten years of individual-based modelling in ecology: what have we learned and what could we learn in the future? Ecol. Model. 115, 129-148

49 Stouffer, D.B. (2010) Scaling from individuals to networks in food webs. Funct. Ecol. 24, 44-51

50 Thompson, R.M. and Townsend, C.R. (2005) Food-web topology varies with spatial scale in a patchy environment. Ecology 86, 1916-1925

51 Hassell, M.P. et al. (1991) Spatial structure and chaos in insect population-dynamics. Nature $353,255-258$

52 Amarasekare, P. et al. (2004) Mechanisms of coexistence in competitive metacommunities. Am. Nat. 164, 310-326

53 Loeuille, N. (2010) Consequences of adaptive foraging in diverse communities. Funct. Ecol. 24, 18-27

54 Loreau, M. et al. (2003) Biodiversity as spatial insurance in heterogeneous landscapes. Proc. Natl. Acad. Sci. U.S.A. 100, 1276512770

55 Thompson, R.M. and Townsend, C.R. (1999) The effect of seasonal variation on the community structure and food-web attributes of two streams: implications for food-web science. Oikos 87, 75-88

56 Tavares-Cromar, A.F. and Williams, D.D. (1996) The importance of temporal resolution in food web analysis: Evidence from a detritusbased stream. Ecol. Monogr. 66, 91-113

57 Petchey, O.L. et al. (1999) Environmental warming alters food-web structure and ecosystem function. Nature 402, 69-72

58 Woodward, G. et al. (2010) Ecological networks in a changing climate. Adv. Ecol. Res. Ecol. Netw. 42, 71-138

59 Harley, C.D.G. (2011) Climate change, keystone predation and biodiversity loss. Science $334,1124-1127$

60 Tylianakis, J.M. et al. (2008) Global change and species interactions in terrestrial ecosystems. Ecol. Lett. 11, 1351-1363

61 Cattin, M.F. et al. (2004) Phylogenetic constraints and adaptation explain food-web structure. Nature 427, 835-839

62 Bolnick, D.I. et al. (2011) Why intraspecific trait variation matters in ecology. Trends Ecol. Evol. 26, 183-192

63 McKane, A.J. (2004) Evolving complex food webs. Eur. Phys. J. B. 38, 287-295

64 Loeuille, N. and Loreau, M. (2005) Evolutionary emergence of sizestructured food webs. Proc. Natl. Acad. Sci. U.S.A. 102, 5761-5766

65 Schluter, D. (2009) Evidence for ecological speciation and its alternative. Science 323, 737-741

66 The Marie Curie SPECIATION Network (2011) What do we need to know about speciation? Trends Ecol. Evol. 27, 27-39
67 Pachepsky, E. et al. (2007) Consequences of intraspecific variation for the structure and function of ecological communities. Part 2. Linking diversity and function. Ecol. Model. 207, 277-285

68 Post, D.M. et al. (2000) Prey preference by a top predator and the stability of linked food chains. Ecology 81, 8-14

69 Hubbell, S.P. (2001) The Unified Neutral Theory of Biodiversity and Biogeography, Princeton University Press

70 Srivastava, D.S. and Vellend, M. (2005) Biodiversity-ecosystem function research: Is it relevant to conservation? Annu. Rev. Ecol. Evol. Syst. 36, 267-294

71 Pocock, M.J.O. et al. (2012) The robustness and restoration of a network of ecological networks. Science 335, 973-977

72 Thebault, E. and Fontaine, C. (2010) Stability of ecological communities and the architecture of mutualistic and trophic networks. Science 329, 853-856

73 Sahasrabudhe, S. and Motter, A.E. (2011) Rescuing ecosystems from extinction cascades through compensatory perturbations. Nat. Commun. 2, 170

74 Saavedra, S. et al. (2011) Strong contributors to network persistence are the most vulnerable to extinction. Nature 478, 233-235

75 Coll, M. et al. (2008) Structural degradation in Mediterranean Sea food webs: testing ecological hypotheses using stochastic and mass-balance modelling. Ecosystems 11, 939-960

76 Duffy, J.E. et al. (2007) The functional role of biodiversity in ecosystems: incorporating trophic complexity. Ecol. Lett. 10, 522-538

77 Duffy, J.E. (2003) Biodiversity loss, trophic skew and ecosystem functioning. Ecol. Lett. 6, 680-687

78 Petchey, O.L. et al. (2008) Trophically unique species are vulnerable to cascading extinction. Am. Nat. 171, 568-579

79 Ives, A.R. and Cardinale, B.J. (2004) Food-web interactions govern the resistance of communities after non-random extinctions. Nature 429 , 174-177

80 Branch, T.A. et al. (2010) The trophic fingerprint of marine fisheries. Nature 468, 431-435

81 Williams, R.J. and Martinez, N.D. (2008) Success and its limits among structural models of complex food webs. J. Anim. Ecol. 77, 512-519

82 Stouffer, D.B. et al. (2006) A robust measure of food web intervality. Proc. Natl. Acad. Sci. U.S.A. 103, 19015-19020

83 Zook, A.E. et al. (2011) Food webs: Ordering species according to body size yields high degree of intervality. J. Theor. Biol. 271, 106-113

84 Laliberte, E. and Legendre, P. (2010) A distance-based framework for measuring functional diversity from multiple traits. Ecology 91, 299305

85 Wiegert, R.G. (1975) Simulation models of ecosystems. Ann. Rev. Ecol. Syst. 6, 311-338

86 Coll, M. et al. (2011) Food-web structure of seagrass communities across different spatial scales and human impacts. PLOS ONE 6, e22591

87 Townsend, C.R. et al. (1998) Disturbance, resource supply, and foodweb architecture in streams. Ecol. Lett. 1, 200-209

88 O'Dowd, D.J. et al. (2003) Invasional 'meltdown' on an oceanic island. Ecol. Lett. 6, 812-817

89 Cross, W.F. et al. (2011) Ecosystem ecology meets adaptive management: food web response to a controlled flood on the Colorado River, Glen Canyon. Ecol Appl. 21, 2016-2033

90 Tylianakis, J. et al. (2007) Habitat modification alters the structure of tropical host-parasitoid food webs. Nature 455, 202-205

91 Warren, P.H. (1994) Making connections in food webs. Trends Ecol. Evol. 9, 136-141

92 Martinez, N.D. (1992) Constant connectance in community food webs. Am. Nat. 139, 1208-1218

93 Schoener, T.W. (1989) Food webs from the small to the large. Ecology 70, 1559-1589

94 Williams, R.J. and Martinez, N.D. (2000) Simple rules yield complex food webs. Nature 404, 180-183

95 Closs, G. et al. (1993) Constant predator prey ratios - an arithmetical artifact. Ecology 74, 238-243

96 Dunne, J.A. et al. (2002) Network structure and biodiversity loss in food webs: robustness increases with connectance. Ecol. Lett. 5, 558-567

97 Yoon, I. et al. (2004) Webs on the Web (WoW): 3D visualization of ecological networks on the WWW for collaborative research and education. Proceedings of the IS\&T/SPIE Symposium on Electronic Imaging, Visualization and Data Analysis, Volume 5295, pp. 124-132 